\title{
Ex situ Lift Out of PFIB Prepared TEM Specimens
}

\author{
Lucille A. Giannuzzi ${ }^{1}$ and Noel S. Smith ${ }^{2}$ \\ 1. EXpressLO LLC, Fort Myers, FL USA. \\ 2. Oregon Physics, LLC, Hillsboro, OR USA.
}

Inductively coupled plasma sources are capable of producing $\mathrm{Xe}^{+}$ions for commercial focused ion beam (FIB) applications [1,2]. It should be no surprise that the longstanding $\mathrm{Ga}^{+} \mathrm{FIB}$ strategies for transmission electron microscopy (TEM) specimen preparation are directly applicable and transferrable to the plasma FIB (PFIB). Indeed, we showed a few years ago that the $\mathrm{Xe}^{+}$PFIB can produce electron transparent specimens for TEM where lift out is not required [3]. Advantages to $\mathrm{Xe}^{+}$ions are (i) they are inert and therefore will not react adversely with the specimen; and (ii) they are heavier than $\mathrm{Ga}^{+}$ions and thus stopped closer to the surface, thereby reducing the depth of implantation damage at similar energies [3-5]. The use of high current $\mathrm{Xe}^{+}$ions allows for large TEM specimens to be prepared within reasonable time frames.

New developments in ex situ lift out called EXpressLO ${ }^{\mathrm{TM}}$ combine fast, easy, and reproducible site specific specimen preparation with the flexibility and capability for further processing [6-8]. In particular, the EXpressLO ${ }^{\mathrm{TM}}$ grid negates the need for a carbon film, allowing for post manipulation FIB, broad ion milling, or plasma cleaning if needed. Moreover, eliminating the carbon film allows for flexible TEM analysis. EXpressLO ${ }^{\mathrm{TM}}$ also provides for quick and easy manipulation into a backside configuration for reducing FIB milling curtaining artifacts [7]. A single ex situ lift out system can support multiple FIB instruments and is therefore cost effective.

In this paper, we combine PFIB specimen preparation of suitable lift out type specimens with ex situ lift out and manipulation using EXpressLO ${ }^{\mathrm{TM}}$. The high beam currents available with the PFIB are advantageous for fast milling of large specimens. FIG. 1a shows PFIB prepared trenches containing a 1 $\mu \mathrm{m}$ thick specimen that is $\sim 50 \mu \mathrm{m} \times 20 \mu \mathrm{m}$ in size and completely PFIB milled free. Note that this is more than twice the size of a conventional $\mathrm{Ga}^{+}$ion prepared TEM specimen. The PFIB time required to prepare this large specimen was quick at $<13$ minutes. FIG. 1a shows the glass manipulator needle positioned and ready to perform the specimen lift out. FIG. 1b shows the specimen lifted out from its trench and attached to the glass needle using just adhesion forces. FIG. 1c shows the manipulation of the PFIB prepared specimen to an EXpressLO ${ }^{\mathrm{TM}}$ grid. The lift out and manipulation steps are performed in just a few minutes. If the specimen was electron transparent it could be taken directly to the TEM for analysis [8]. At this point the specimen can be taken back to an SEM or other instrument for analysis [9]. Alternatively, the specimen could be put back into the $\mathrm{Xe}^{+}$PFIB or $\mathrm{Ga}^{+}$FIB for further thinning [68]. Thus, combining $\mathrm{Xe}^{+}$PFIB and ex situ lift out for the preparation of TEM specimens increases throughput and allows for larger specimens to be analyzed.

\section{References:}

[1] N.S. Smith et al., J. Vac. Sci. Technol. B 246 (2006) p. 2902.

[2] Paul Tesch et al., ISTFA Conference Proceedings, ASM International (2008) p.7.

[3] L.A. Giannuzzi and N.S. Smith, Microsc. Microanal., 17 S2 (2011) p. 646

[4] R.D. Kelly et al., Microsc. Microanal. 19 S2 (2013) p. 862. 
[5] N. Bassim, K. Scott, and L.A. Giannuzzi, to be published, MRS Bulletin (2014)

[6] L.A. Giannuzzi, Microsc. Microanal. 18 (S2) (2012) p. 632.

[7] L.A. Giannuzzi, Proc. ISTFA (2012) p. 388.

[8] L.A. Giannuzzi, Microsc. Microanal. 19 (S2) (2013) p. 906.

[9] F.A. Stevie et al., Surf. Interface Anal. 31 (2001) p. 345.

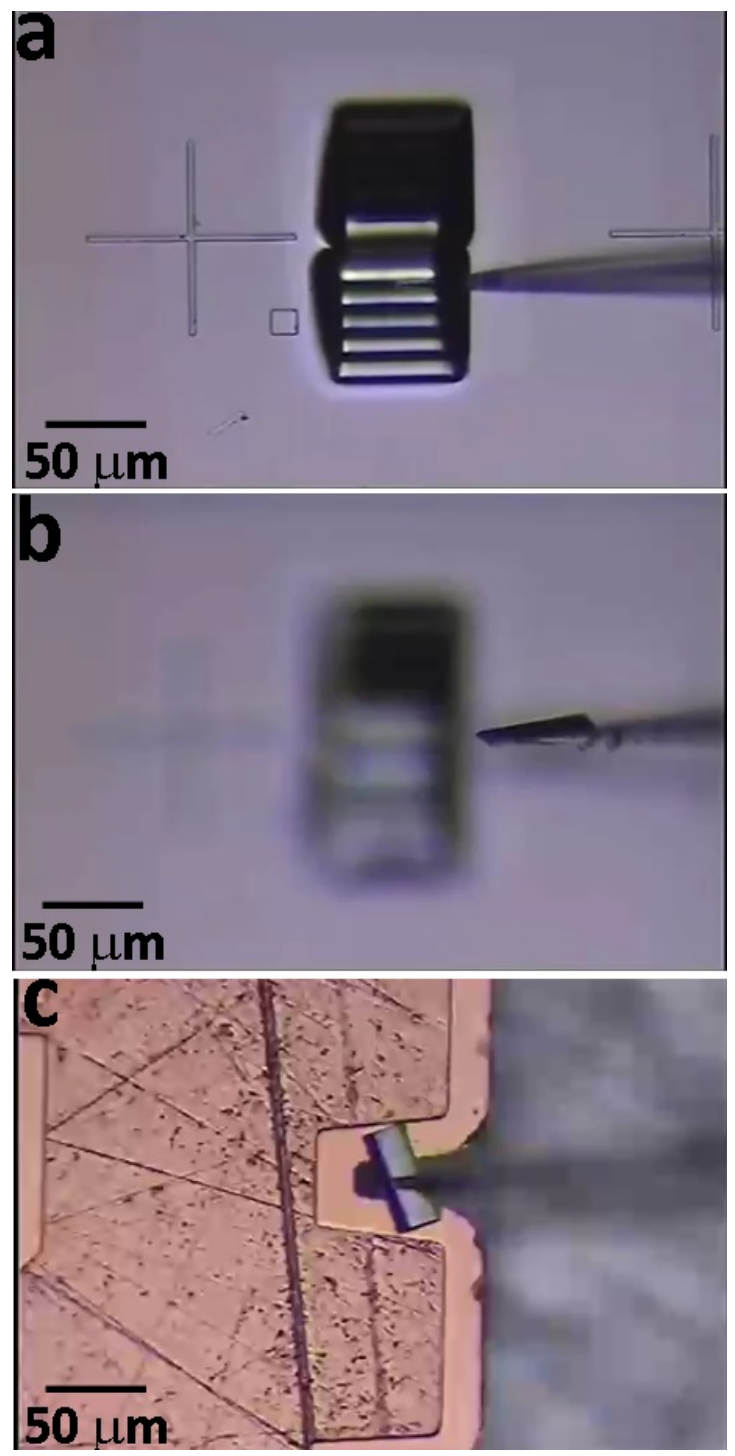

Figure 1. ex situ lift out of a $50 \mu \mathrm{m}$ long PFIB prepared TEM specimen. (a) The micromanipulator needle nears the PFIB specimen for lift out. (b) The PFIB specimen is attached to the needle. (c) The PFIB specimen is manipulated to an EXpressLO ${ }^{\mathrm{TM}}$ grid using ex situ lift out. 